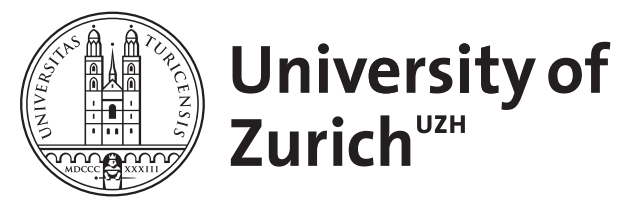

\title{
Wachstumsstörungen als Leitsymptom
}

Thiel, C ; Rauch, A

\begin{abstract}
Kleinwuchs als Leitsymptom stellt eine häufige Fragestellung sowohl in der humangenetischen als auch in der pädiatrischen Sprechstunde dar. Definiert ist Kleinwuchs als eine Körperhöhe unter der 3.Perzentile der Norm bzw. unter -2Standardabweichungen. Diese macht sich bemerkbar durch Änderungen der Wachstumsgeschwindigkeit oder des Wachstumsverlaufs, welche grundsätzlich genetisch determiniert, jedoch auch von sekundären Faktoren beeinflussbar sind. Das Spektrum der zugrunde liegenden genetischen Ursachen reicht von Störungen der Wachstumshormonsekretion und -wirkung über Skelettdysplasien bis hin zu komplexen Fehlbildungssyndromen. Die genetische Abklärung stellt somit einen Grundpfeiler zur Beurteilung der Prognose und einer möglichen therapeutischen Intervention dar. Es werden die grundlegenden diagnostischen Überlegungen anhand häufiger Differenzialdiagnosen, deren genetischen Grundlagen und Behandlungsmöglichkeiten aufgeführt: Ullrich-Turner-Syndrom, Léri-WeillSyndrom, Silver-Russell-Syndrom, Noonan-Syndrom und Achondroplasie
\end{abstract}

DOI: https://doi.org/10.1007/s11825-012-0331-2

Posted at the Zurich Open Repository and Archive, University of Zurich

ZORA URL: https://doi.org/10.5167/uzh-156532

Journal Article

Published Version

Originally published at:

Thiel, C; Rauch, A (2012). Wachstumsstörungen als Leitsymptom. Medizinische Genetik, 24(2):123-137. DOI: https://doi.org/10.1007/s11825-012-0331-2 
Punkte sammeln auf ...

\section{springermedizin.de/}

\section{eAkademie}

\section{Teilnahmemöglichkeiten}

Diese Fortbildungseinheit steht Ihnen als e.CME und e.Tutorial in der Springer Medizin e.Akademie zur Verfügung.

- e.CME: kostenfreie Teilnahme im Rahmen des jeweiligen Zeitschriftenabonnements

- e.Tutorial:Teilnahme im Rahmen des e.Med-Abonnements

\section{Zertifizierung}

Diese Fortbildungseinheit ist mit 3 CMEPunkten zertifiziert von der Landesärztekammer Hessen und der Nordrheinischen Akademie für Ärztliche Fort- und Weiterbildung und damit auch für andere Ärztekammern anerkennungsfähig.

\section{Hinweis für Leser aus Österreich} Gemäß dem Diplom-Fortbildungs-Programm (DFP) der Österreichischen Ärztekammer werden die in der e.Akademie erworbenen CME-Punkte hierfür 1:1 als fachspezifische Fortbildung anerkannt.

\section{Kontakt und weitere}

\section{Informationen}

Springer-Verlag GmbH

Springer Medizin Kundenservice

Tel. 08007780777

E-Mail: kundenservice@springermedizin.de

\section{CME Zertifizierte Fortbildung}

\author{
C. Thiel ${ }^{1} \cdot$ A. Rauch ${ }^{2}$
}

${ }^{1}$ Humangenetisches Institut, Friedrich-Alexander-Universität Erlangen-Nürnberg, Erlangen

${ }^{2}$ Institut für Medizinische Genetik, Universität Zürich, Schwerzenbach-Zürich

\section{Wachstumsstörungen als Leitsymptom}

\section{Zusammenfassung}

Kleinwuchs als Leitsymptom stellt eine häufige Fragestellung sowohl in der humangenetischen als auch in der pädiatrischen Sprechstunde dar. Definiert ist Kleinwuchs als eine Körperhöhe unter der 3. Perzentile der Norm bzw. unter -2 Standardabweichungen. Diese macht sich bemerkbar durch Änderungen der Wachstumsgeschwindigkeit oder des Wachstumsverlaufs, welche grundsätzlich genetisch determiniert, jedoch auch von sekundären Faktoren beeinflussbar sind. Das Spektrum der zugrunde liegenden genetischen Ursachen reicht von Störungen der Wachstumshormonsekretion und -wirkung über Skelettdysplasien bis hin zu komplexen Fehlbildungssyndromen. Die genetische Abklärung stellt somit einen Grundpfeiler zur Beurteilung der Prognose und einer möglichen therapeutischen Intervention dar. Es werden die grundlegenden diagnostischen Überlegungen anhand häufiger Differenzialdiagnosen, deren genetischen Grundlagen und Behandlungsmöglichkeiten aufgeführt: Ullrich-Turner-Syndrom, Léri-Weill-Syndrom, Silver-Russell-Syndrom, Noonan-Syndrom und Achondroplasie.

\section{Schlüisselwörter}

Wachstumsstörung · Kleinwuchs · Wachstumshormon · Skelettdysplasie $\cdot$ Fehlbildungen 


\title{
Lernziel
}

Wachstum stellt ein grundlegendes Merkmal unseres Organismus dar. Die Ursachen von Störungen der Wachstumsgeschwindigkeit und/oder des Wachstumsverlaufs sind sehr heterogen und betreffen i. d. R. zentrale Mechanismen der hormonellen Steuerung, Zellteilung, Morphogenese und Reifung. Neben primären, meist genetisch determinierten Störungen, spielen sekundäre Effekte systemischer Erkrankungen sowie exogene Einflüsse eine Rolle (z. B. Cushing-Syndrom, Zöliakie, Nahrungsmittelunverträglichkeiten, Fettstoffwechselstörungen, schwere systemische Erkrankungen). Die Prävalenz des Kleinwuchses beträgt 3\%. Trotz dieser Häufigkeit und der für viele Betroffenen weitreichenden gesundheitlichen und gesellschaftlichen Probleme sind die genetischen Ursachen in den meisten Fällen jedoch nicht aufgeklärt.

Nachdem Sie diesen Beitrag gelesen haben,

- kennen Sie wichtige Formen und Ursachen von Wachstumsstörungen, insbesondere in der genetischen Beratung häufigere Syndrome,

- sind Ihnen grundlegende diagnostische Überlegungen vertraut, um die Ätiologie abzuklären,

- können Sie ggf. eine gezieltere Therapie einleiten.

\section{Definition}

Die Körperhöhe ist in der Bevölkerung normalverteilt und stellt ein multifaktorielles Merkmal mit hoher Heritabilität dar. Bezogen auf die Population liegt per Definition ein Kleinwuchs vor, wenn die Körperhöhe unter der 3. Perzentile der Alters- und Geschlechts-Norm bzw. unter -2 Standardabweichungen (SD, „standard deviation“) liegt. Die für die Beurteilung des Wachstums bislang zugrunde gelegten Perzentilenkurven sind populationsspezifisch und zeigen in Europa ein Nord-SüdGefälle. Während für Norddeutschland häufig die auf Dortmunder Daten beruhenden Kurven von Reinken und van Ost zugrunde gelegt werden, scheinen für Süddeutschland die aus Zürich stammenden Prader-Kurven geeigneter [1, 2]. Die von der World Health Organisation (WHO) kürzlich zur Verfügung gestellten Perzentilenkurven demgegenüber stellen Referenzwerte für Kinder mit ethnisch unterschiedlichem Hintergrund dar. Ob die Verwendung dieser panethischen Wachstumskurven oder populationsspezifischer Daten medizinisch sinnvoller ist, ist derzeit noch umstritten und wird an verschiedenen Zentren unterschiedlich gehandhabt.

\section{Einteilung und Ätiologie}

Aufgrund der Normalverteilung und multifaktoriellen Genese der Körperhöhe lässt sich anhand der Körperhöhe der Eltern eine sog. genetische Zielgröße ermitteln:

Genetische Zielgröße des Kindes = [(Körperhöhe Vater + Körperhöhe Mutter $) / 2] \pm 6,5 \mathrm{~cm}$

(für Jungen $+6,5 \mathrm{~cm}$, für Mädchen $-6,5 \mathrm{~cm}$ ).

\section{Growth retardation as a cardinal symptom}

\begin{abstract}
Short stature is a frequently encountered question in both the genetic and the pediatric clinic. The definition of short stature includes a body length/height below the 3rd percentile or of more than two standard deviations below the mean. The velocity of growth as well as growth patterns are fundamentally regulated by genetic factors, but may be modified by secondary effects. The spectrum of short stature syndromes includes disorders of growth hormone secretion or effect, skeletal dysplasias and complex malformation syndromes. Identifying the underlying defect greatly facilitates counseling about prognosis and possible management. The most relevant syndromes in daily clinical routine are discussed here: Turner syndrome, Léri-Weill syndrome, Russel-Silver syndrome, Noonan syndrome, and achondroplasia.
\end{abstract}

\section{Keywords}

Growth retardation $\cdot$ Short stature $\cdot$ Growth hormone $\cdot$ Skeletal dysplasia $\cdot$ Malformations 


\begin{tabular}{|l}
\hline Infobox \\
\hline Radiologische Evaluation bei Verdacht \\
auf Skelettdysplasie: \\
- Linke Hand \\
- Wirbelsäule a.-p. und seitlich \\
- Becken a.-p. \\
- Kniegelenk a.-p. \\
Bei klinischen Auffälligkeiten: \\
- Schädel a.-p. und seitlich \\
- Thorax a.-p. \\
- Arm a.-p. \\
- Fuß oder Sprunggelenk seitlich \\
\hline
\end{tabular}

gerung in der Familie geben. Typisch sind hierbei ein später Eintritt der Pubertät bei dem betroffenen Patienten und anamnestisch bei einem Elternteil sowie ein verzögertes Knochenalter. Diese Form der Wachstumsverzögerung stellt aber eine Ausschlussdiagnose dar, da letztendlich erst ein Aufholwachstum in der Pubertät die Diagnose bestätigt.

Klinisch unterscheidet sich der syndromale Kleinwuchs durch das Vorliegen zusätzlicher Auffälligkeiten oder Fehlbildungen vom nichtsyndromalen. Daneben kann die Wachstumsverminderung mit einer Verschiebung der normalen Körperproportionen einhergehen (disproportionierter Kleinwuchs) und entweder nach der Geburt oder bereits intrauterin beginnen (primordialer Kleinwuchs; „intrauterine growth retardation“, IUGR). Ein disproportionierter Kleinwuchs weist i. d. R. auf eine gestörte Knochenreifung bzw. -differenzierung hin. Man unterscheidet derzeit 456 verschiedene Formen solcher Skelettdysplasien, von denen 316 auf Defekte in 226 bisher bekannten Genen zurückgeführt werden können [3]. Diese lassen sich grundsätzlich klinisch weiter einteilen in Skelettdysplasien mit kurzem Rumpf und weitestgehend unauffälligen Extremitäten bzw. Formen mit deutlich verkürzten Extremitäten bei ansonsten weitestgehend unauffälliger Konfiguration des Rumpfes. Die Verkürzung der Extremitäten kann das obere Segment betreffen (rhizomel, z. B. Achondroplasie), oder das mittlere Segment (mesomel, z. B. mesomele Dysplasie nach Langer), oder Hände und Füße (akromel, z. B. geleophysische Dysplasie).

Neben der Röntgenaufnahme der linken Hand zur Bestimmung des Knochenalters können weitergehende Röntgenaufnahmen eine Differenzierung und Einteilung durch den Nachweis einer Beteiligung der Wirbelkörper (spondylär), der Wachstumsfugen (epiphysär), des Knochenschafts (diaphysär) und/oder des Teils zwischen Epiphyse und Diaphyse (metaphysär) ermöglichen (• Infobox). Skelettdysplasien sind aber selten und treten insgesamt nur bei 0,04\% der Neugeborenen auf [4]. Die häufigste Form ist die unten näher erläuterte Achondroplasie.

Bei proportioniertem Kleinwuchs hingegen sind weitreichende Röntgenaufnahmen selten zielführend. Hierbei können jedoch assoziierte dysmorphologische Merkmale eine Einordnung in ein bekanntes Syndrom ermöglichen (z. B. Ullrich-Turner- oder Noonan-Syndrom). Die wichtigste Differenzialdiagnose des nichtsyndromalen Kleinwuchses stellt der hypophysäre Wachstumshormonmangel dar (• Tab. 1). Mutationen in mehreren Genen sind hierfür als ursächlich beschrieben worden. Eine mögliche Einteilung unterscheidet Defekte der Wachstumshormonbildung durch Mutationen im GH-Gen (17q22-q24) selbst, Mutationen in Regulatoren der Wachstumshormonsekretion (z. B. PROP1-Gen (5q35)), oder eine Wachstumshormoninsensitivität verursacht durch Mutationen im GH-Rezeptor(GHR)-Gen (17q11.2, Laron-Syndrom, MIM \#262500). Diese sind i. d. R. autosomal-rezessiv vererbt und die Diagnose erfolgt im Rahmen der pädiatrischen endokrinologischen Abklärung. Ein Wachstumshormonmangel liegt bei etwa 1:4000-10.000 Kindern vor, wobei sich in etwa 70\% der Fälle weder eine sekundär-organische noch eine primäre Ursache nachweisen lässt. Durch die Gabe von rekombinantem Wachstumshormon steht eine effektive Therapie zur Verfügung. Nach Ausschluss eines Wachstumshormonmangels bleibt die Ätiologie der nichtsyndromalen Wachstumsverzögerung oft ungeklärt (idiopathischer Kleinwuchs).

Bei über $80 \%$ der Betroffenen mit nicht hormonell begründetem Kleinwuchs kann keine ätiologisch begründete Diagnose gestellt werden $[5,6,7]$. Der Anteil der syndromalen Formen kann auf etwa 3\% und der chromosomaler Veränderungen (z. B. Ullrich-Turner-Syndrom) auf etwa 15\% geschätzt werden (• Tab. 2).
Syndromaler Kleinwuchs geht mit zusätzlichen Auffälligkeiten oder Fehlbildungen einher

Die Verkürzung der Extremitäten kann das obere Segment betreffen (rhizomel), das mittlere (mesomel) oder Hände und Füße (akromel)

Röntgenaufnahmen zeigen eine spondyläre, epi-, dia- oder metaphysäre Beteiligung 
Tab. 1 Endokrinopathien als Ursache einer Wachstumsstörung

\begin{tabular}{lll}
\hline Erkrankung & Gen & Vererbung \\
\hline Laron-Syndrom & GHR & AR \\
\hline Kombinierter Hypophysenhormonmangel & PROP 1 & AR \\
\hline Hypopituitarismus & SOX3 & XLR \\
\hline IGF1-Mangel & IGF1 & AR \\
\hline IGF1-Resistenz & IGF1R & AR, AD \\
\hline Isolierter Wachstumshormonmangel & GH1 & AR, AD \\
\hline AD
\end{tabular}

AD autosomal-dominant; $A R$ autosomal-rezessiv; $X L R$ „X-linked recessive", $X$-chromosomal-rezessiv.

Tab. 2 Häufige Ursachen syndromaler Wachstumsstörung

\begin{tabular}{llll} 
Erkrankung & Gen/Locus & Vererbung & Häufigkeit \\
\hline Noonan-Syndrom & $\begin{array}{l}\text { TPN11, SOS 1, KRAS, NRAS, } \\
\text { RAF1, BRAF, SHOC2 und } \\
\text { MEK1/MAP2K1 }\end{array}$ & AD & $1: 1000-1: 2500$ \\
\hline Ullrich-Turner-Syndrom & Xp & Sporadisch & $1: 2500$ \\
\hline SHOX-Gen-Defekte & SHOX & XLD & $1: 4000$ \\
\hline DiGeorge-Syndrom & Mikrodeletion 22q11.2 & AD & $1: 4000$ \\
\hline Silver-Russell-Syndrom & UPD7, Chr. 11p15 & Sporadisch & $1-30: 100.000$ \\
\hline Prader-Willi-Syndrom & 15q11-q13 & Sporadisch & $1: 10.000-1: 30.000$ \\
\hline Coffin-Lowry-Syndrom & RPS6KA3 & XLD & $1: 40.000$ \\
\hline Kabuki-Syndrom & MLL2 & AD & $1: 32.000-1: 86.000$ \\
\hline $\begin{array}{l}\text { Trichorhinophalangeales } \\
\text { Syndrom }\end{array}$ & TRPS1 & AD & Unklar \\
\hline AD autosomal-dominant; XLD „X-linked dominant“, X-chromosomal-dominant. &
\end{tabular}

Tab. 3 Checkliste zur Abklärung von Wachstumsverzögerungen

\begin{tabular}{|ll}
\hline Anamnese & Schwangerschaftsverlauf (Wachstumsverzögerung in der Schwangerschaft) \\
\cline { 2 - 2 } & Geburtsparameter: Größe, Gewicht, Kopfumfang (KU) \\
\cline { 2 - 2 } Familienanamnese & Geburtsmaße und Auxiologie der Eltern und Geschwister \\
\hline Körperliche Untersuchung & Körperlänge/-höhe, Gewicht, KU, Armspanne, Sitzhöhe, Körperproportionen \\
\cline { 2 - 2 } & Skelettauffälligkeiten (Rhizomelie/Mesomelie, Einschränkung der Beweglichkeit) \\
\hline Kraniofaziale Dysmorphien \\
\hline Diagnostik & $\begin{array}{l}\text { Endokrinologische Diagnostik (Wachstumshormon, Schilddrüsenhormon), Stoff- } \\
\text { wechseldiagnostik, Antikörperdiagnostik (Anti-Gliadin-AK) }\end{array}$ \\
\hline Röntgendiagnostik bei V. a. Skelettdysplasie \\
\hline ggf. Echokardiographie und Sonographie der Nieren \\
\hline ggf. Entwicklungsdiagnostik \\
\hline ggf. Chromosomenanalyse mit FISH-Analyse z. A. Ullrich-Turner-Syndrom \\
\hline ggf. spezifische molekulargenetische Analysen \\
\hline
\end{tabular}

\section{Auxiologie}

Zur Abgrenzung möglicher Ursachen und zur Beurteilung, inwieweit eine Wachstumsverzögerung vorliegt, müssen neben der Anamnese und der Körperhöhe weitere Parameter erhoben werden (• Tab. 3). Grundsätzlich erfolgt die Bestimmung der Körperlänge bei Kindern bis 2 Jahren im Liegen und danach der Körperhöhe im Stehen. Neben den aktuellen Parametern sind besonders die Geburtsmaße und der Verlauf des Wachstums zu erheben. Die Wachstumsgeschwindigkeit unter Einbezug der Messwerte über einen längeren Zeitraum ermöglicht hier die weitere Eingrenzung der Wachstumsstörung. Insbesondere Kinder, die „small for gestational age“ (SGA) geboren wurden und bis zum 4. Lebensjahr kein Aufholwachstum zeigen, sowie Kinder, die im Lauf ihres Wachstums mehrere Perzentilen nach unten überkreuzen, sollten weiter untersucht werden.

Zur Beurteilung der Proportionen sind außerdem die Messung des Kopfumfangs, des Gewichts, der Armspanne und der Sitzhöhe notwendig. Darüber hinaus kann es im Einzelfall sinnvoll sein, auch 


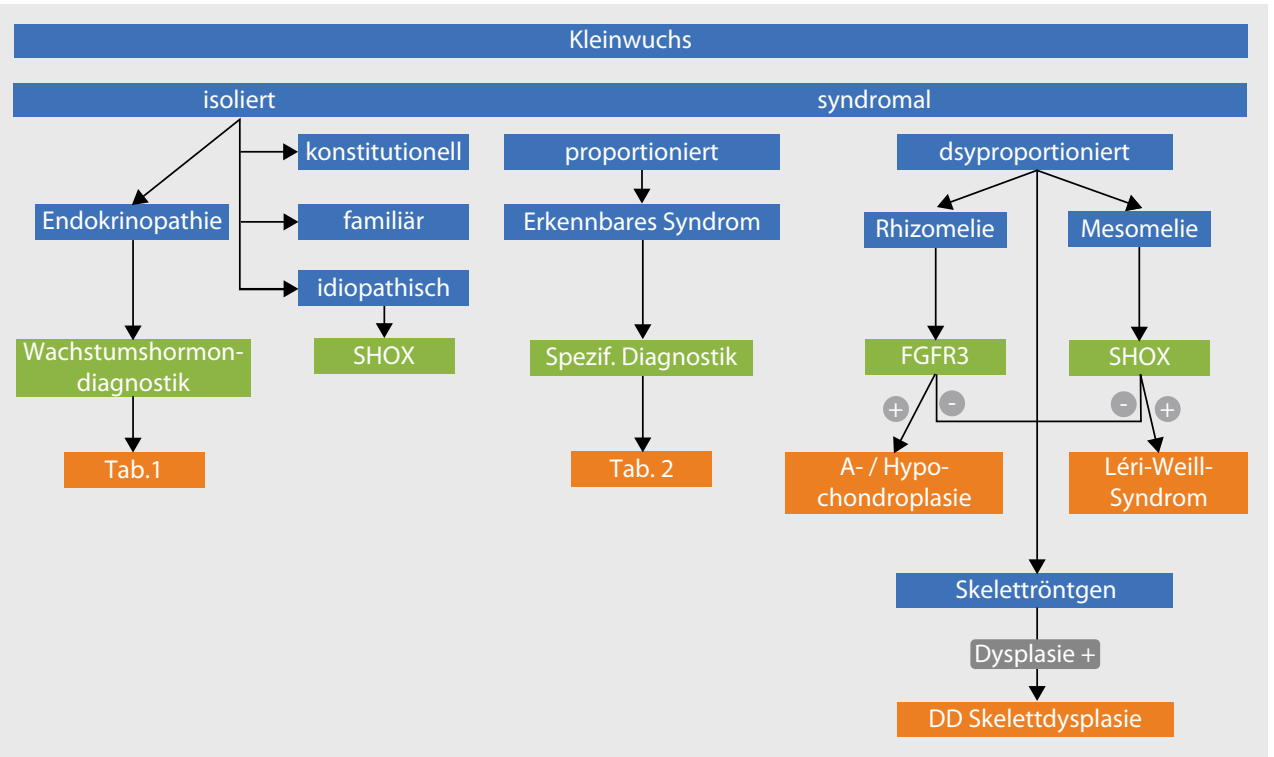

Abb. $1 \Delta$ Einteilung und diagnostisches Vorgehen bei Kleinwuchs

die Beinlänge oder Pubishöhe und die Hand- und Fußlänge zu erheben. Normwerttabellen für die verschiedenen Parameter finden sich z. B. bei Hall et al. [8]. Das Verhältnis der Oberarm- zu Unterarmlänge bzw. Oberschenkel- zu Unterschenkellänge ist i. d. R. nur anhand von Röntgenbildern bei Kindern sicher zu bestimmen, kann aber im Rahmen der körperlichen Untersuchung abgeschätzt werden. Die Bestimmung des Knochenalters erfolgt zur Evaluation der Knochenreife. Allerdings ist selbst ein signifikant verzögertes Knochenalter ein unspezifischer Faktor und tritt bei unterschiedlichen Ursachen der Wachstumsverzögerung auf.

Aufgrund ihrer Häufigkeit und Relevanz in der genetischen Beratung wird auf einzelne Krankheitsbilder im Folgenden näher eingegangen. Die $\bullet$ Abb. 1 gibt einen Überblick zum generellen diagnostischen Vorgehen.

\section{Ullrich-Turner-Syndrom}

Das Ullrich-Turner-Syndrom (UTS) ist die häufigste Chromosomenaberration bei Mädchen und Frauen und kommt mit einer Häufigkeit von 1:2500 bei weiblichen Lebendgeburten vor. Dieses Syndrom, erstmals von Otto Ullrich 1930 beschrieben und 1938 von Henry Turner als eigenständiges Syndrom etabliert, umfasst die Kriterien Wachstumsstörung, Pterygium colli, „Schildthorax“, Überstreckbarkeit der Ellenbogengelenke und eine ausbleibende Geschlechtsentwicklung. In etwa 60\% liegt dem UTS der Verlust eines X-Chromosoms durch eine meiotische Non-Disjunction mit einem Karyotyp 45,X0 vor. Ein Mosaik zwischen einer 45,X0- und einer 46,XX-Zelllinie liegt demgegenüber in etwa 6-20\% vor. Diese Angaben unterscheiden sich in der Literatur allerdings wesentlich. Daneben gibt es auch teilweise Mosaike mit 3 Zelllinien, 46,X0/46,XX/47,XXX, oder mit einer 46,XYZelllinie. Zum Nachweis geringgradiger Mosaike kann eine Interphasen-Fluoreszenz-in situ-Hybridisierungs(FISH)-Analyse an Lymphozyten und/oder Mundepithelzellen oder eine Chromosomenund FISH-Analyse an einer Ovarialbiopsie notwendig sein. Neben diesen numerischen Aberrationen können einem UTS auch strukturelle Aberrationen des X-Chromosoms, wie z. B. Isochromosom Xq, Ringchromosom oder Deletionen eines X-Chromosoms, in allen Zellen oder als Mosaike zugrunde liegen. Diese verschiedenen Formen können zur Variabilität des Phänotyps beitragen. Patientinnen, bei denen in einer Zelllinie Y-chromosomale Anteile nachgewiesen werden können, weisen ein auf über 30\% erhöhtes Gonadoblastomrisiko auf. Methodisch ist dieser Nachweis sowohl durch FISHAnalysen als auch durch die molekulargenetische Analyse z. B. der SRY-Region möglich. Bei diesen Patientinnen oder bei solchen mit reinem 45,X-Karyotyp, bei denen eine Y-Zelllinie nicht sicher ausgeschlossen werden kann, sollte nach der Pubertät eine Ovarialbiopsie oder die Entfernung der Eierstöcke in Erwägung gezogen werden.

Das Ullrich-Turner-Syndrom ist die häufigste Chromosomenaberration bei Mädchen 

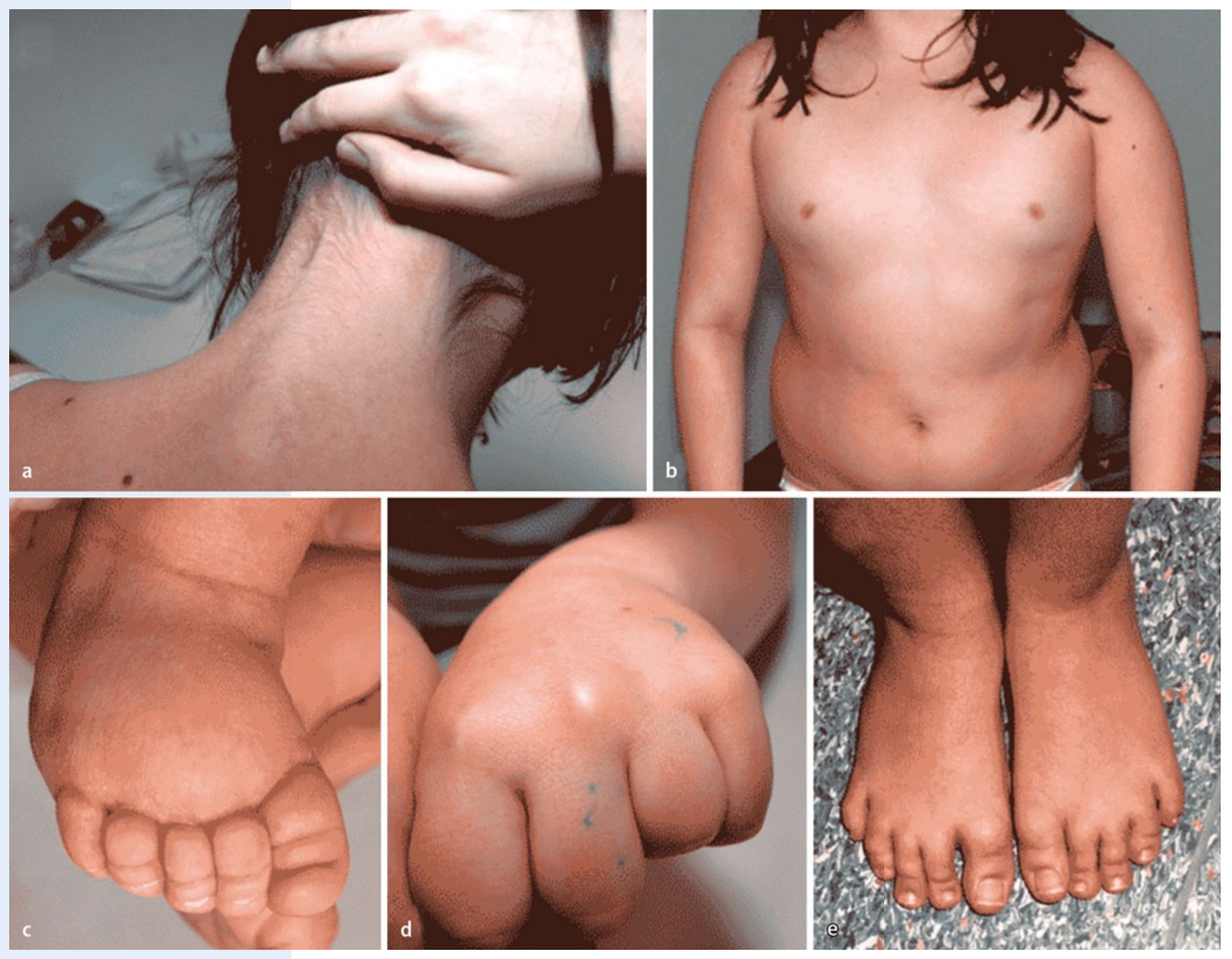

Abb. $2 \Delta$ Charakteristische klinische Zeichen des Ullrich-Turner-Syndroms. a Tiefer, invertierter Haaransatz, b breiter Thorax, c Fußrückenödeme nach Geburt, $\mathbf{d}$ Brachymetakarpie IV und V

\section{Häufige Fehlbildungen sind \\ Herzfehler}

Die wesentlichsten Probleme, die mit dem UTS einhergehen, sind der Kleinwuchs und später eine Unfruchtbarkeit aufgrund einer Gonadendysgenesie. Die mittlere Körperhöhe im Erwachsenenalter liegt bei unbehandelten Patientinnen mit UTS bei etwa 142-147 cm. Zwölf Prozent der Patientinnen liegen in einem Bereich $\pm 12 \mathrm{~cm}$ [9] der genannten Werte. Zur Beurteilung stehen UTS-spezifische Perzentilenkurven zur Verfügung. Die Gonadendysgenesie führt zur primären oder sekundären Amenorrhö, und es gibt nur wenige Einzelberichte über Schwangerschaften bei Frauen mit UTS, die kein erkennbares Mosaik mit einer normalen Zelllinie haben. Die Strukturen von Gebärmutter und Scheide sowie das äußere Genitale sind normal angelegt, die Geschlechtsorgane bleiben aufgrund des Hormonmangels unbehandelt jedoch präpubertär. Häufige Fehlbildungen sind Herzfehler, welche bei $40-59 \%$ der Patientinnen vorliegen, insbesondere Aortenisthmusstenosen, eine bikuspide Aortenklappe oder Aortenaneurysmen. Zu den in etwa 40-59\% der Fälle beobachteten Nierenfehlbildungen zählen Hufeisennieren, Nierenaplasien und Nierenbecken- bzw. Harnleiteranomalien. Neben den typischen kleinen Anomalien wie kurzer, breiter Hals mit tiefem, invertiertem Haaransatz, Pterygium colli, Epikanthus und hoher Gaumen sind Mittelohrentzündungen, Zahnfehlstellungen und Skelettanomalien wie Brachymetakarpie IV oder Skoliose häufiger ( $\bullet$ Abb. 2). Die neonatal bei 60-79\% der Patientinnen beobachteten Lymphödeme der Hand- und Fußrücken bessern sich i. d. R. im Verlauf der Kindheit, können aber auch persistieren. Etwas gehäuft findet man bei Patientinnen mit UTS Störungen der Schilddrüsenfunktion mit Nachweis von Schilddrüsenautoantikörpern. Die geistige Entwicklung verläuft bei Mädchen mit UTS meist normal, bei 8-11\% liegt jedoch eine Intelligenzminderung vor, die v. a. auf visuell-räumliche und sprachliche Defizite zurückgeführt wird. 

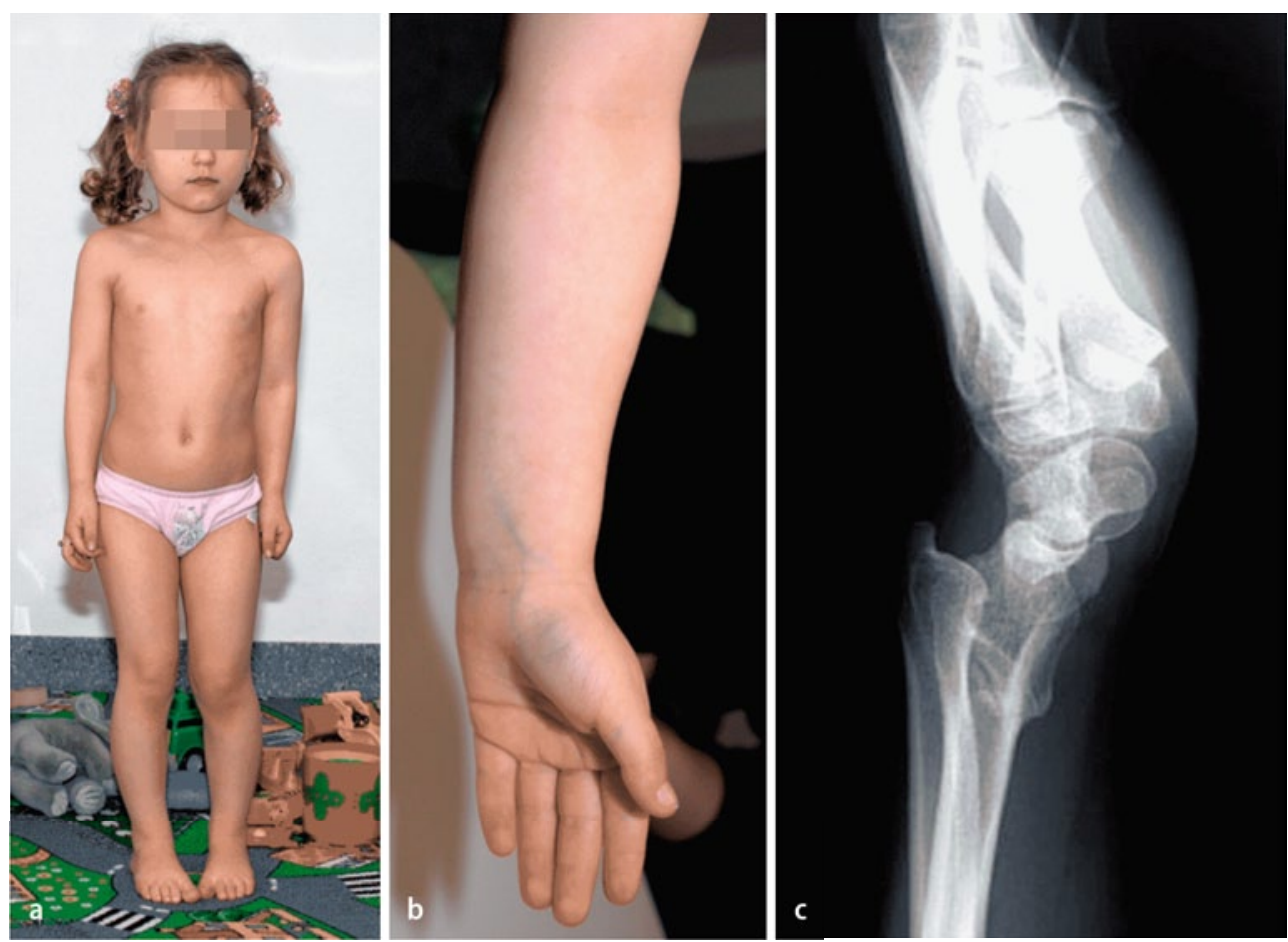

Abb. 3 A Klinische Merkmale der Léri-Weill-Dyschondrosteosis aufgrund eines SHOX-Gendefekts. a Disproprtionierter Kleinwuchs mit Mesomelie. b, c Mesomelie und Madelung-Deformität

Differenzialdiagnostisch ist hier besonders das Noonan-Syndrom zu nennen, bei dem der Herzfehler allerdings bevorzugt die rechte Seite mit einer Pulmonalstenose als Kardinalsymptom betrifft. Patienten mit Noonan-Syndrom weisen häufiger eine psychomotorische Entwicklungsverzögerung auf und i. d. R. keine Gonadendysgenesie.

Die Behandlung des UTS ist der Symptomatik entsprechend komplex, wobei zur Behandlung des hypergonadotropen Hypogonadismus und der Wachstumsstörung die effektivsten therapeutischen Möglichkeiten bestehen [10]. In fast allen Fällen ist eine Östrogensubstitution zur Feminisierung im Rahmen der Pubertät notwendig. Erfolgt die Geschlechtshormonbehandlung weitestgehend altersgerecht, ermöglicht sie einen normalen Verlauf der Pubertät und senkt zudem das Osteoporoserisiko. Die Behandlung mit rekombinantem Wachstumshormon stellt das Mittel der Wahl zur Behandlung der Wachstumsstörung dar [11]. Nach frühzeitigem Beginn der Wachstumshormongabe erreichen bis zu etwa 63\% der Patientinnen eine Endgröße über -2 SD [12].

\section{Erkrankungen durch SHOX-Gen-Defekte}

Das SHOX-Gen ist in der pseudoautosomalen Region der X- und Y-Chromosomen lokalisiert und entgeht der X-Inaktivierung. Die SHOX-Haploinsuffizienz führt zu einer variablen Ausprägung von einer isolierten Wachstumsverzögerung über die Léri-Weill-Dyschondrosteosis (MIM \#127300, LWD) bis zur mesomelen Dysplasie nach Langer (MIM \#249700, LMD) bei Betroffenheit beider Allele. Die Prävalenz der SHOX-Haploinsuffizienz wird auf etwa 1:1000 geschätzt [13] und stellt somit in etwa 2-4\% die Ursache einer Wachstumsverzögerung dar.

Die LWD ist charakterisiert durch die Leitsymptome Kleinwuchs, Mesomelie und Madelung-Deformität (- Abb. 3). Der Kleinwuchs ist dabei disproportioniert und progressiv. Betroffene Kinder liegen bei der Geburt mit etwa -1,05 SD im Normbereich. Während der Kindheit verlangsamt sich das Wachstum und die Endgröße liegt durchschnittlich bei $-2,8$ SD für Frauen und $-2,36$ SD bei Männern [14]. Dies wird insbesondere auf einen verminderten Pubertätswachstumsschub zurückgeführt. Die Verkürzung der Unterarme (Mesomelie) wird hervorgerufen durch eine reduzierte Radiuslänge im Rahmen der Madelung-Deformität und entwickelt sich in variablen Ausmaß erst im Laufe der Kindheit. Die nach Langer definierten radiologischen Kriterien für eine Madelung-Deformität umfassen neben der Verkürzung des Radius eine dreieckige Form der distalen radialen Epiphy- 
Tab. 4 Diagnostische Kriterien beim Silver-Russell-Syndrom

\begin{tabular}{|c|c|}
\hline \multirow[t]{4}{*}{ Hauptkriterien („major criteria“) } & Intrauterine Wachstumsverzögerung ( $<10$. Perzentile) \\
\hline & Postnatale Wachstumsverzögerung ( $<3$. Perzentile) \\
\hline & Normaler Kopfumfang (relative Makrozephalie) \\
\hline & Körperasymmetrie \\
\hline \multirow[t]{4}{*}{ Nebenkriterien („minor criteria“) } & Verringerte Armspanne, proportioniert \\
\hline & Klinodaktylie V \\
\hline & Trianguläres Gesicht \\
\hline & Prominente Stirn \\
\hline \multirow[t]{5}{*}{ Unterstützende Kriterien } & Café-au-lait-Flecken, Pigmentflecken \\
\hline & Genitale Anomalien \\
\hline & Psychomotorische Entwicklungsverzögerung \\
\hline & Ernährungsprobleme \\
\hline & Hypopglykämie \\
\hline
\end{tabular}

se (Spitze des Dreiecks zeigt nach medial; „triangularization“), eine Verkeilung der Handwurzelknochen zwischen dem deformierten Radius und der protrahierten Ulna, wodurch sich eine Dreiecksform mit dem Os lunatum an der Spitze ergibt („pyramidaliszation“), eine Transparenzerhöhung der distalen radialen Metaphyse („lucency“) sowie eine Dislokation oder Subluxation des distalen Radioulnargelenks, wobei die distale Ulna hinter dem distalen Radius steht. Die Madelung-Deformität kann zu einer Einschränkung der Pro- und Supination führen. Auch die Unterschenkel können betroffen sein. Aufgrund der möglichen Unterarm- und/oder Unterschenkeldeformität können belastungsabhängige Schmerzen entstehen, die dann orthopädischer Therapie bedürfen. Bei der mesomelen Dysplasie nach Langer liegt aufgrund eines biallelischen Defekts eine ausgeprägtere Skelettdysplasie mit einer Endgröße von etwa $130 \mathrm{~cm}$ vor.

Ursächlich liegen der SHOX-Haploinsuffizienz pseudoautosomal-dominant vererbte Deletionen oder Punktmutationen des SHOX-Gens zugrunde. Heterozygote Deletionen oder Punktmutationen werden bei $2-15 \%$ der Patienten mit ,idiopathischem“ Kleinwuchs und bei $50-100 \%$ der Patienten mit LWD nachgewiesen $[15,16]$. Deletionen unterschiedlicher Größe der SHOX-Genregion liegen bei etwa 70\% der Patienten mit einer SHOX-Defizienz vor. In etwa der Hälfte betreffen diese Deletionen bei Patienten mit LWD nicht das SHOX-Gen selbst, sondern die zentromer gelegenen Enhancer-Regionen [17]. Punktmutationen im SHOX-Gen als Ursache werden in 5-30\% nachgewiesen (SHOX Allelic Variant Database: http://www.hd-lovd.uni-hd.de/). Genetische Ursache der LMD sind homozygote Deletionen oder compound-heterozygote Mutationen des SHOX-Gens, gewöhnlich bei Nachkommen mit LWD. Die Diagnostik der SHOX-Defizienz sollte i. d. R. mittels „multiplex ligation-dependent probe amplification“ (MLPA) erfolgen, da die FISH-Analyse kleinere Deletionen oder Deletionen in den Enhancer-Bereichen nicht erfasst. Lässt sich keine Deletion nachweisen, ist eine Punktmutationsanalyse mittels Sequenzierung indiziert. Auch bei der SHOX-Defizienz kann die Endgröße ähnlich wie beim Ullrich-Turner-Syndrom durch die Gabe von rekombinantem Wachstumshormon verbessert werden.

\section{Silver-Russell-Syndrom}

Das Silver-Russell-Syndrom (MIM \#180860) wurde 1953 von Russell und Silver als Kombination der Symptome intrauterine (primordialer Kleinwuchs) und postnatale Wachstumsverzögerung, relative Makrozephalie (Pseudohydrozephalus), häufig Körperasymmetrien und Klinodaktylie V beschrieben. Das klinische Bild ist sehr variabel und keines der Merkmale ist obligat [18, 19, 20]. Die Haupt- und Nebenkriterien sind in $\bullet$ Tab. 4 zusammengefasst. Die klinische Diagnose ergibt sich, wenn 3 Hauptkriterien oder 2 Hauptkriterien und 2 Nebenkriterien vorliegen.

Die Häufigkeit in der Bevölkerung wird mit etwa 1-30 auf 100.000 Neugeborene angegeben. ÄuBerliche Charakteristika umfassen eine - meist pränatal beginnende - stark reduzierte Körperhöhe mit reduziertem Gewicht aufgrund geringen subkutanen Fettgewebes und ein hierzu disproportioniert, aber altersentsprechend normal großer Kopf. Dies führt bei manchen Betroffenen zusammen mit einem triangulären Gesicht zu dem Eindruck eines Pseudohydrozephalus (• Abb. 4). Ernährungsprobleme mit ausgeprägter Gedeihstörung machen im 1. Lebensjahr oft eine Sondenernährung 
Abb. 4 Charakteristische Fazies beim Silver-Russell-Syndrom

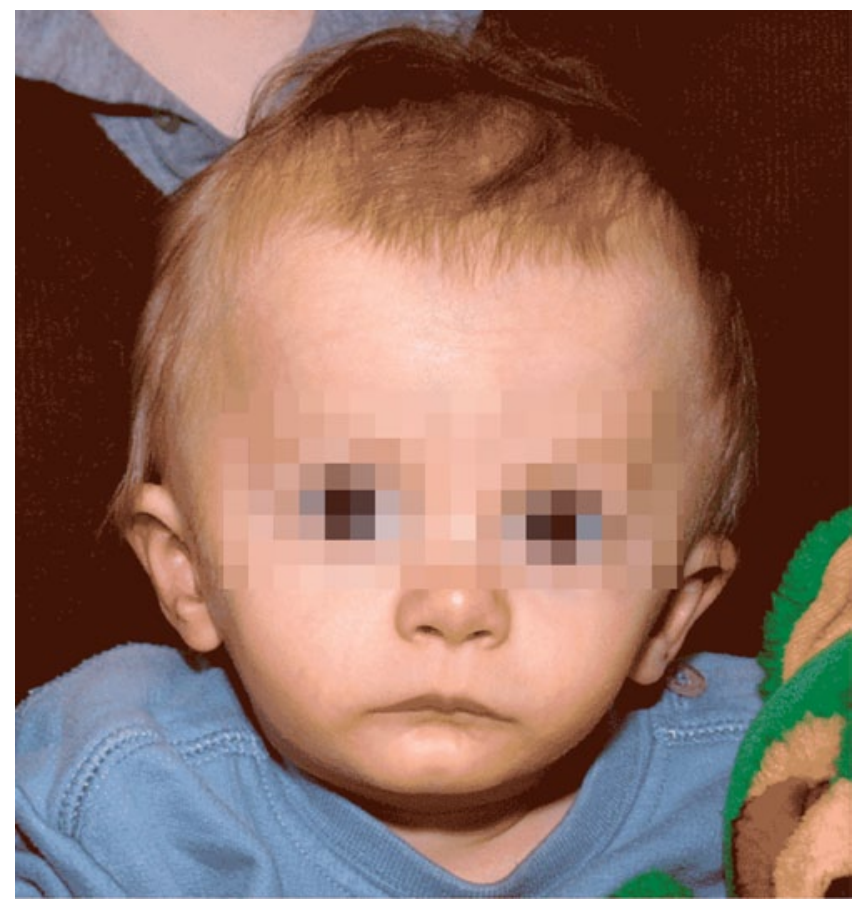

notwendig. In 60-80\% der Fälle besteht eine leichte Asymmetrie der Gliedmaßen (Hemihypertrophie). Häufiger sind auch gastrointestinale Störungen wie z. B. Reflux oder eine Neigung zu Hypoglykämien. Das Körperwachstum ist trotz einer leicht verminderten Armspanne i. d. R. proportioniert. Die durchschnittliche Endgröße beim Silver-Russell-Syndrom ohne Hormonbehandlung wird mit ungefähr $151 \mathrm{~cm}$ für Männer und mit etwa $140 \mathrm{~cm}$ für Frauen angegeben. Für die Beurteilung des Wachstums liegen für das Silver-Russell-Syndrom eigene Perzentilenkurven vor [21]. Die psychomotorische Entwicklung von Kindern mit einem Silver-Russell-Syndrom ist bei dem größten Teil der Patienten normal. Obwohl die motorischen Meilensteine sich bei etwa 34\% der Patienten leicht verspätet entwickeln, holen die Kinder diesen Rückstand bis zum Schulalter i. d. R. wieder auf. Verzögerungen der mentalen Entwicklung, insbesondere der Sprachentwicklung, sind bei Silver-RussellSyndrom-Patienten mit 20-65\% häufig aber eher gering ausgeprägt [22]. Eine Behandlung ist symptomorientiert. Aufgrund der nicht eindeutigen Abgrenzung des Krankheitsbildes ist das Ansprechen auf eine Wachstumshormonbehandlung ohne Nachweis der zugrunde liegenden Ursache nicht sicher vorherzusagen. Patienten mit molekulargenetisch nachgewiesenem Silver-Russell-Syndrom auch ohne Wachstumshormonmangel scheinen aber von einer Behandlung zu profitieren [23]. Auch wenn hier im Detail noch keine ausreichenden Daten über die Effektivität dieser Behandlung in Abhängigkeit der zugrunde liegenden Ursache vorliegen, besteht auf Grundlage der intrauterinen Wachstumsverzögerung ohne Aufholwachstum eine Indikation zur Behandlung.

Das Silver-Russell-Syndrom ist genetisch heterogen. Die zwei bekannten Ursachen sind die maternale uniparentale Disomie 7 (UPD7) und Veränderungen einer differenziell methylierten Region auf Chromosom 11p15.5. Die Region 11p11.5 unterliegt einem genomischen Imprinting durch Methylierung zweier imprinting center (ICs) innerhalb von 2 "imprinting control regions" (ICRs). Die epigenetische Modifikation einer telomerwärts gelegenen ICR1 führt zu einer monoallelischen Expression der Gene $H 19$ (nichtkodierende RNA, paternal methyliert, maternal exprimiert) und des fetalen Wachstumsfaktors IGF2 (,,insulin-like growth factor 2", maternal methyliert, paternal exprimiert). Die Modifikation der zentromerwärts gelegenen ICR2 führt zur monoallelischen Expression von KCNQ1 (maternal exprimiert), KCNQ10T1 (paternal exprimiert) und CDKN1C (maternal exprimiert). Während eine Hypomethylierung des IC1 und IC2 auf dem maternalen Chromosom zu dem Beckwith-Wiedemann-Großwuchssyndrom führt, findet sich bei 30-50\% der Patienten mit Silver-Russell-Syndrom eine Hypomethylierung des IC1 auf dem paternalen Allel und somit eine biallele Expression des H19-Gens und eine biallele Repression des IGF2-Gens [24]. In seltenen Fällen wurden auch genomische Duplikationen der maternalen 11p15.5 Region bei Silver-Russell-Patienten beobachtet. Die genauen Mechanismen sind allerdings noch ungeklärt. Eine maternale UPD7 wird 


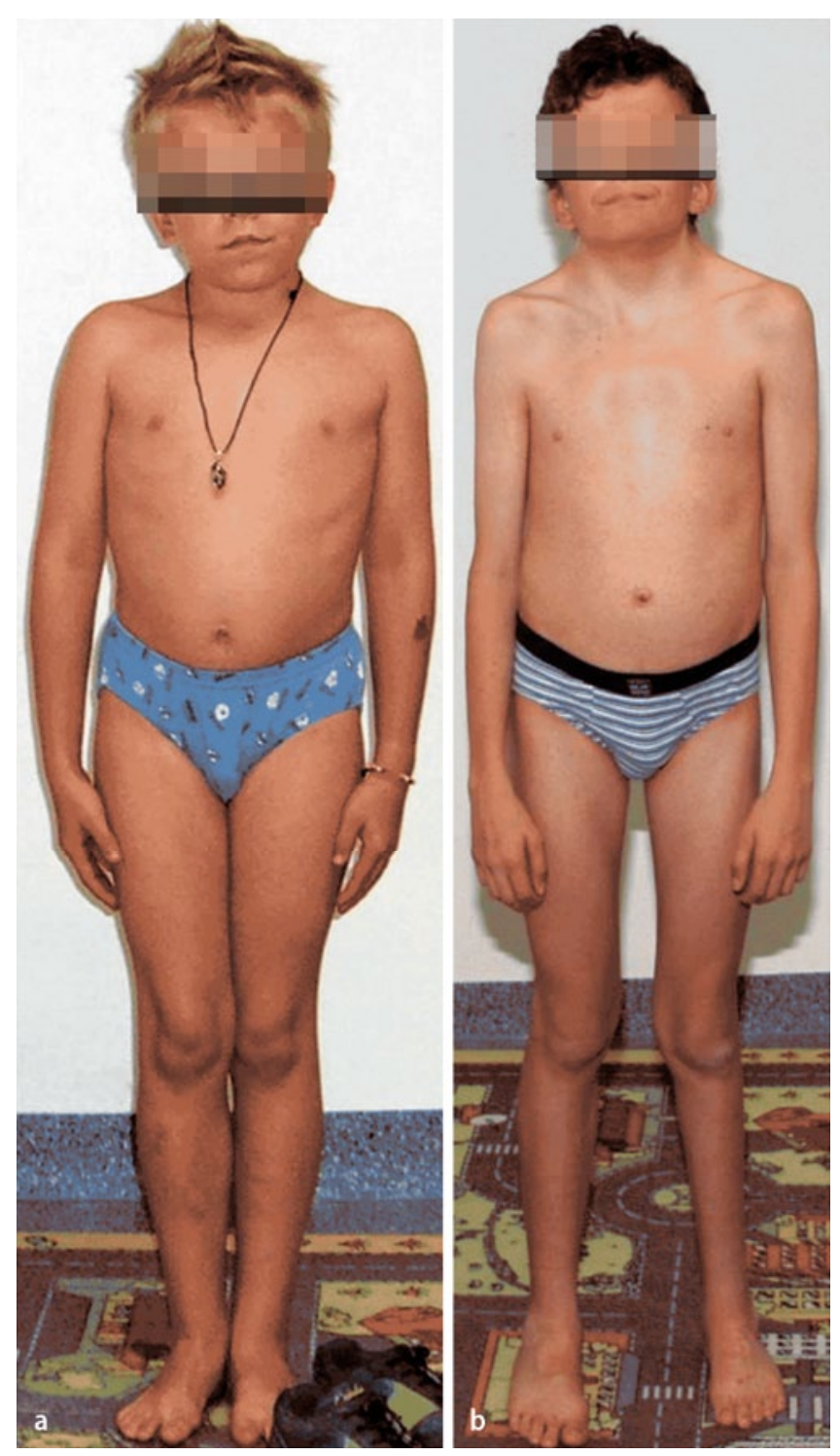

Abb. $5<$ Klinische Merkmale des Noonan-Syndroms mit breitem Thorax und leichter Sternumdeformität

\section{Das Noonan-Syndrom ist eine variable Kombination von postnatalem Klein- wuchs, angeborenem Herzfehler und äußeren Merkmalen wie z. B. fazialen Dysmorphien}

bei bis zu 10\% der Patienten beobachtet. Die genaue beteiligte Region auf Chromosom 7 ist nicht bekannt. In einzelnen Fällen wurden Deletionen 7q21 und Duplikationen 7p11.2-p12 mit einem SilverRussell-Phänotyp beobachtet. Bei etwa 30\% der Patienten mit dem Verdacht auf ein Silver-RussellSyndrom bleibt allerdings die genetische Ursache unklar. Retrospektive Untersuchungen ergaben keinen Unterschied im Phänotyp zwischen Patienten mit einer mUPD7 und einer Hypomethylierung 11p15.5. Die ICR1-Hypomethylierung scheint aber in der Mehrzahl eine intrauterine und postnatale Wachstumsverzögerung und eine Asymmetrie zu beinhalten. Eine leichte psychomotorische Entwicklungsverzögerung scheint häufiger bei Patienten mit einer mUPD7 (65\%) gegenüber Patienten mit einer 11p15-Hypomethylierung (20\%) zu sein. Aufgrund der Variabilität des Phänotyps sollte auch bei Vorliegen einzelner Merkmale eine Diagnostik durchgeführt werden.

\section{Noonan-Syndrom}

Das Noonan-Syndrom wurde erstmals 1968 von Jacqueline Noonan als variable Kombination von postnatalem Kleinwuchs, angeborenem Herzfehler und einigen typischen äußeren Merkmalen, wie weiter Augenabstand, absteigende Lidachsen, Ptosis, tief angesetzte Ohren und Brustkorbdeformitäten, beschrieben ([25], - Abb. 5). Die Inzidenz liegt bei etwa 1:1000-1:2500. Der Phänotyp ist sehr variabel und wird mit zunehmendem Alter unspezifischer. Die diagnostischen Kriterien nach van der Burgt sind in $\bullet$ Tab. 5 dargestellt. Die Diagnose Noonan-Syndrom kann klinisch gestellt wer- 
Tab. 5 Diagnostische Kriterien für das Noonan-Syndrom

\begin{tabular}{|lll}
\hline Merkmal & A & B \\
\hline Fazial & Typische faziale Dysmorphien & Hinweisende faziale Dysmorphien \\
\hline Herz & $\begin{array}{l}\text { Pulmonalstenose, HOCM und/oder typi- } \\
\text { sche EEG-Veränderungen }\end{array}$ & Andere Herzfehler \\
\hline Körperhöhe & $<$ 3. Perzentile & $<10$. Perzentile \\
\hline Brustwand & Pectus carinatum/excavatum & Breiter Thorax \\
\hline Familienanamnese & Erstgradig Verwandter mit NS & Erstgradig Verwandter mit vermutetem NS \\
\hline Weitere & $\begin{array}{l}\text { Entwicklungsverzögerung, Kryptorchismus } \\
\text { und lymphatische Dysplasie }\end{array}$ & $\begin{array}{l}\text { Entweder Entwicklungsverzögerung oder } \\
\text { Kryptorchismus oder lymphatische Dys- } \\
\text { plasie }\end{array}$ \\
\hline $\begin{array}{l}\text { A Major-, B Minor-Kriterium, EEG Elektroenzephalogramm, HOCM hypertrophe obstruktive Kardiomyopathie, } \\
\text { NS Noonan-Syndrom. }\end{array}$ & \\
\hline
\end{tabular}

den, wenn 2 Major-Kriterien, ein Major-Kriterium und 2 Minor-Kriterien oder wenn 4 Minor-Kriterien vorliegen.

Bei den angeborenen Herzfehlern (50-80\%) handelt es sich am häufigsten (20-50\%) um eine Pulmonalstenose, gefolgt von der hypertrophen Kardiomyopathie (20-30\%) und Vorhofscheidewanddefekten. Die Häufigkeit der Kardiomyopathie unterscheidet sich in Abhängigkeit vom Gendefekt und liegt bei Mutationen im RAF1-Gen bei 75-95\%. Gewicht und Körperlänge sind bei Geburt meist im Normbereich und verlaufen bis zur Pubertät parallel zur 3. Perzentile der Norm. Das Knochenalter ist verzögert und die Pubertät setzt verspätet ein, wobei der pubertäre Wachstumsschub meist ausbleibt oder vermindert ist. Die mittlere Erwachsenengröße für Männer mit NoonanSyndrom wird mit etwa 162,5 cm;, die von Frauen mit 152,7 cm angegeben. Letztendlich liegen etwa 50\% der Frauen und etwa 40\% der Männer von ihrer Erwachsenengröße unterhalb des Normbereichs. Eine vergrößerte Nackenfalte im 1. Trimenon stellt ein häufiges Zeichen (20\%) einer Dysplasie der Lymphgefäße dar. Neben einem Pterygium colli als Restzustand des fetalen Ödems bei Geburt können auch periphere, pulmonale und intestinale Lymphangiektasien vorliegen. Bei etwa 55\% der Patienten mit Noonan-Syndrom kann eine leichte Blutungsneigung vorliegen. Diese ist aber nur in etwa 3\% der Fälle klinisch relevant. Vor einer eventuellen Operation sollte aber der Gerinnungsstatus genau geprüft werden.

Die psychomotorische Entwicklung betroffener Kinder kann verzögert sein, auch wenn die Mehrzahl der Kinder die Regelschule besucht. Eine leichte motorische Verzögerung der Meilensteine im ersten Lebensjahr wird auf die muskuläre Hypotonie und die Gelenküberstreckbarkeit zurückgeführt. Etwa 25\% weisen eine Lernschwäche und etwa 10-15\% eine moderate Retardierung auf [26, 27]. Es handelt sich v. a. um Verzögerungen in der Sprachentwicklung. Insgesamt ist der klinische Ausprägungsgrad des Noonan-Syndroms sehr variabel. Unter vielen anderen möglichen Symptomen findet sich eine mittel- oder innenohrbedingte Schwerhörigkeit bei 15-40\% der Patienten.

Das Noonan-Syndrom ist genetisch heterogen. Pathogenetisch liegen dem Noonan-Syndrom autosomal-dominant vererbte Gain-of-function-Mutationen in Genen des Ras-MAPK-Signalwegs zugrunde. Bislang wurden Mutationen in den Genen PTPN11, SOS1, KRAS, NRAS, RAF1, BRAF, SHOC2 und MEK1/MAP2K1 bei Patienten mit Noonan-Syndrom nachgewiesen. Keimbahnmutationen in einiger dieser Gene und weiterer Gene des Ras-MAPK-Signalwegs wurden bei Krankheitsbildern mit überlappenden Phänotypen beschrieben, z. B. Costello-Syndrom, kardiofaziokutanes Syndrom, Neurofibromatose Typ 1 oder Legius-Syndrom. Mit 50\% sind Mutationen im PTPN11Gen die häufigste Ursache des Noonan-Syndroms, gefolgt von SOS1 (10-13\%) und RAF1 (3-17\%).

Leitlinien für die Behandlung von Patienten mit Noonan-Syndrom wurden u. a. von der europäischen Noonan Syndrome Development Group erarbeitet (http://www.dyscerne.org/dysc/digitalAssets/0/265_Noonan_Guidelines.pdf). Ein Ansprechen des Wachstums auf Wachstumshormonbehandlung auch bei Noonan-Patienten ohne nachweisbaren Wachstumshormonmangel scheint mit einer Änderung der Endgröße von $+0,6$ bis $+1,8$ SD gegeben zu sein [28].

\section{Achondroplasie}

Die erstmals von Parrot 1878 erwähnte Achondroplasie (MIM \#100800) gehört mit einer Prävalenz von 1:15.000 bis 1:40.000 zu den häufigsten Chondrodysplasien. Die wichtigsten klinischen Charak- 

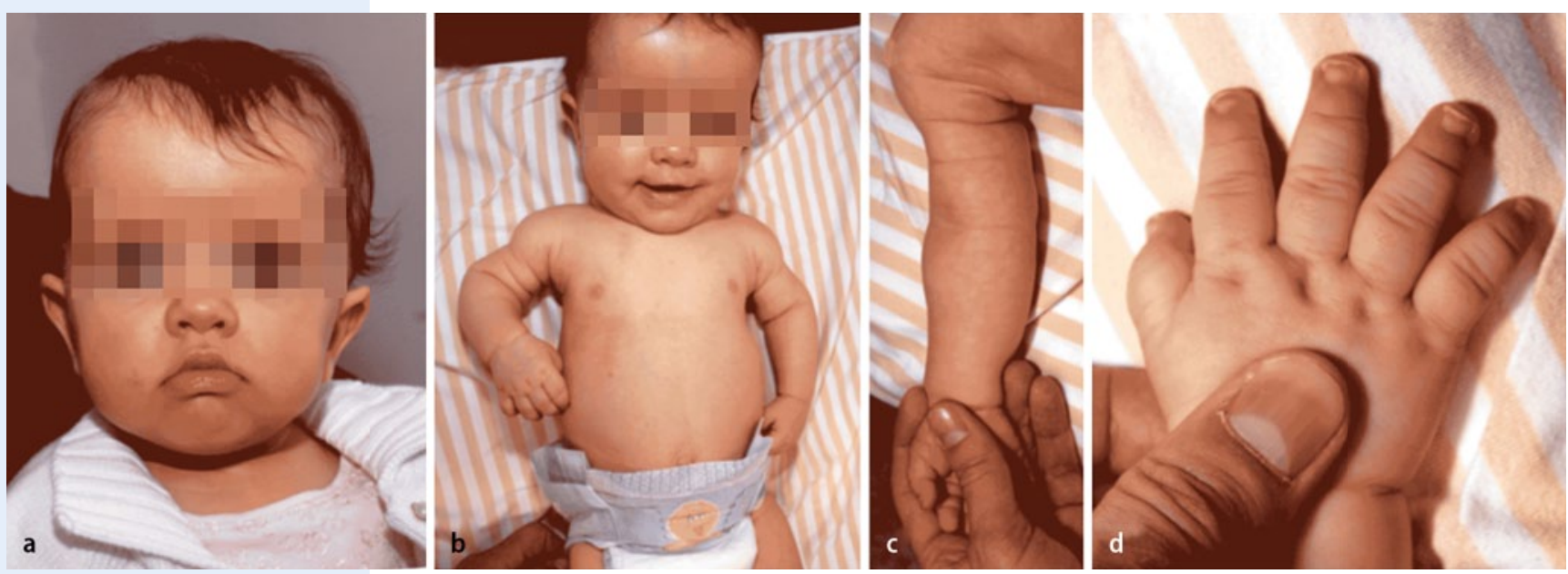

Abb. $6 \Delta$ Charakteristische klinische Zeichen der Achondroplasie. a, b Faziale Gestalt mit relativer Makrozephalie, prominenter Stirn und Mittelgesichtshypoplasie. b, c Rhizomelie der Arme. d Dreizackstellung der Finger

Wichtige Charakteristika der Achondroplasie sind disproportionierter Kleinwuchs mit rhizomeler Verkürzung der Arme und Beine, relative Makrozephalie, prominente Stirn und Mittelgesichtshypoplasie teristika sind ein disproportionierter Kleinwuchs mit rhizomeler Verkürzung der Arme und Beine, eine relative Makrozephalie, faziale Charakteristika mit prominenter Stirn und Mittelgesichtshypoplasie, eine Streckhemmung im Ellenbogengelenk, eine Hyperlordose der Lendenwirbelsäule und eine Dreizackstellung der Finger ([29], - Abb. 6). Die Disproportionierung der Extremitäten, insbesondere eine Verkürzung der Femora, ist gelegentlich bereits pränatal, ansonsten bei Geburt nachweisbar. Das Längenwachstum ist insgesamt deutlich beeinträchtigt bei ansonsten weitestgehend normaler Wirbelsäulenlänge und Kopfumfang. Bei Frauen liegt die Endgröße i. d. R. im Mittel bei $124 \pm 5,9 \mathrm{~cm}$ und bei Männern im Mittel bei 131 $\pm 5,6 \mathrm{~cm}$. Eine Wachstumshormonbehandlung ergibt zwar zu Beginn eine erhöhte Wachstumsgeschwindigkeit, hat aber keinen Einfluss auf die Endgröße, sodass diese Behandlung nicht empfohlen wird. Es besteht jedoch die Möglichkeit, bei Jugendlichen mit Achondroplasie die Endgröße durch eine operative Bein- und Armverlängerung zu erhöhen. Die Intelligenz ist bei Achondroplasie primär normal, jedoch ist aufgrund der körperlichen Disproportion die motorische Entwicklung im 1. Lebensjahr meist verzögert. Deshalb ist bei Kindern mit Achondroplasie generell eine physiotherapeutische Förderung zu empfehlen.

Ein weiteres häufiges Problem stellen Verengungen des Formen magnum und/oder des Rückenmarkkanals dar. Einerseits kann es hierdurch zu einem behandlungsbedürftigen Hydrozephalus kommen, andererseits können neurologische Probleme in Form von zentralen Apnoen, Parästhesien oder Paresen auftreten. Bei 4,7\% der unter 6 Monate alten Säuglinge treten Apnoen auf, jedoch muss nur bei $0,5 \%$ eine operative zervikomedulläre Entlastung erfolgen. Wegen dieser Problematik und des verhältnismäßig großen Kopfes sollte darauf geachtet werden, dass der Kopf nicht zu abrupt bewegt wird. Im Lauf des Lebens treten behandlungsbedürftige Atempausen mit zunehmendem Alter häufiger auf und werden bei $16 \%$ der über 20-Jährigen beobachtet. Rückenschmerzen und neurologische Symptome der Beine treten i. d. R. erst im Lauf der Zeit auf und werden bei über 50-Jährigen mit Achondroplasie in 70-80\% der Fälle gefunden. Das gleiche gilt für die hierfür ursächliche Einengung des Rückenmarkkanals, welche bei $18 \%$ der über 40-Jährigen nicht operationsbedürftig und bei $24 \%$ operationsbedürftig ist. Wegen eines Hydrozephalus oder neurologischer Probleme benötigen 2\% der Säuglinge eine Shuntoperation, im weiteren Verlauf benötigen dies 6-10\% der Patienten. Die Atmung kann allerdings auch wegen eines etwas engen Thorax oder einer bronchialen Obstruktion behindert sein. Ein weiteres Problem können häufige Mittelohrentzündungen darstellen, die zu einer Hörminderung und damit auch zu einem verzögerten Spracherwerb führen können. Auch Zahn- und Kieferfehlstellungen kommen häufiger vor und sollten besondere Beachtung finden. Aus diesen Merkmalen ergeben sich auch mögliche Komplikationen im Rahmen einer Narkose, über die die Patienten/Eltern aufgeklärt werden sollten. Hierbei ist auch auf ein leicht erhöhtes Risiko für eine maligne Hyperthermie hinzuweisen. Für die ausführliche Informationen zur Vorsorge und Behandlung einer Achondroplasie liegen Empfehlungen der „AAP Committee on Genetics“ vor [30].

Dominante Mutationen im FGFR3-Gen („fibroblast growth factor receptor 3“) sind die einzige bekannte Ursache der Achondroplasie. In 98\% der Fälle liegt die rekurrente Mutation c.1138 G > A (p.Gly380Arg) vor. Weitere Mutationen im FGFR3-Gen sind selten. Mutationen im FGFR3-Gen 
führen neben der Achondroplasie auch zu dem geringer ausgeprägten Phänotyp der Hypochondroplasie und der letalen thanatophoren Dysplasie [31]. Bei über 80\% der Patienten liegt eine De-novo-Mutation vor.

\section{Chromosomale Aberrationen und syndromale Wachstumsverzögerung}

Neben den Krankheitsbildern, bei denen die Wachstumsstörung den zentralen Bestandteil des Phänotyps ausmacht, ist die Wachstumsstörung auch nebenbefundlich ein häufiges Merkmal von Mikrodeletions-/Duplikations-Syndromen. Die hier meist im Vordergrund stehenden dysmorphologischen Merkmale und die Entwicklungsverzögerung ermöglichen in vielen Fällen die Formulierung einer Verdachtsdiagnose.

\section{Fazit für die Praxis}

- Kommt es zur Verschiebung der normalen Körperproportionen, handelt es sich um disproportionierten Kleinwuchs. Primordialer Kleinwuchs beginnt bereits intrauterin.

- Durch Röntgenaufnahmen ist festzustellen, ob Wirbelkörper (spondylär), Wachstumsfugen (epiphysär), Knochenschaft (diaphysär) oder der Bereich zwischen Epiphyse und Diaphyse (metaphysär) betroffen sind.

- Die wichtigste Differenzialdiagnose des nichtsyndromalen Kleinwuchses ist der hypophysäre Wachstumshormonmangel. Dieser kann durch Defekte der Wachstumshormonbildung oder eine Wachstumshormoninsensitivität verursacht sein.

- Beim syndromalen Kleinwuchs bestehen weitere Auffälligkeiten oder Fehlbildungen, nicht aber beim nichtsyndromalen Kleinwuchs.

- Zum Ullrich-Turner-Syndrom gehören Wachstumsstörung, Pterygium colli, ,,Schildthorax“, Überstreckbarkeit der Ellenbogengelenke und ausbleibende Geschlechtsentwicklung; zumeist liegt der Karyotyp 45,X0 vor.

- Leitsymptome der Léri-Weill-Dyschondrosteosis sind disproportionierter, progressiver Kleinwuchs, Mesomelie und Madelung-Deformität.

- Das Silver-Russell-Syndrom weist ein klinisch variables Bild auf. Dazu können folgende Symptome gehören: intrauterine und postnatale Wachstumsverzögerung, relative Makrozephalie, Körperasymmetrien und Klinodaktylie V. Als Ursachen sind eine maternale uniparentale Disomie 7 oder genomisches Imprinting einer differenziell methylierten Region auf Chromosom 11p15.5 bekannt.

- Beim Noonan-Syndrom besteht eine variable Kombination von postnatalem Kleinwuchs, angeborenem Herzfehler und äußeren Merkmalen, z. B. weiter Augenabstand, absteigende Lidachsen, Ptosis, tief angesetzte Ohren und Brustkorbdeformitäten. Zugrunde liegen autosomal-dominant vererbte Gain-of-function-Mutationen in Genen des Ras-MAPK-Signalwegs.

- Typisch für die Achondroplasie sind disproportionierter Kleinwuchs, rhizomele Verkürzung der Arme und Beine, relative Makrozephalie, prominente Stirn, Mittelgesichtshypoplasie, Streckhemmung im Ellenbogengelenk, Hyperlordose der Lendenwirbelsäule und Dreizackstellung der Finger. Ursächlich sind dominante Mutationen im FGFR3-Gen.

\section{Korrespondenzadresse}

\section{A. Rauch}

Institut für Medizinische Genetik, Universität Zürich

Schorenstr. 16, 8603 Schwerzenbach-Zürich

Anita.rauch@medgen.uzh.ch

Danksagung. Wir danken allen Patienten und Eltern für ihr Einverständnis zur Veröffentlichung der Fotos. Außerdem bedanken wir uns bei Helmuth-Günther Dörr und Martin Zenker für ihre Kooperation und hilfreichen kritischen Diskussionen.

Interessenkonflikt. Der korrespondierende Autor weist für sich und seine Koautoren auf folgende Beziehung(en) hin: Von Norvartis gesponserte Studie zur Behandlung des fragilen X-Syndroms. 


\section{Literatur}

1. Reinken L, Oost G van (1992) Longitudinal physical development of healthy children 0-18 years of age. Body length/height, body weight and growth velocity. Klin Padiatr 204:129-133

2. Prader A et al (1988) Physical growth of Swiss children from birth to 20 years of age: first Zürich longitudinal study of growth and development. Helv Paediatr Acta Suppl 52:1125

3. Warman ML et al (2011) Nosology and classification of genetic skeletal disorders: 2010 revision. Am J Med Genet A 155A:943-968

4. Kallen B et al (1993) Monitoring dominant germ cell mutations using skeletal dysplasias registered in malformation registries: an international feasibility study. Int J Epidemiol 22:107-115

5. Seaver LH, Irons M (2009) ACMG practice guideline: genetic evaluation of short stature. Genet Med 11:465-470

6. Ahmed ML et al (1993) Evaluation of a district growth screening programme: the Oxford growth study. Arch Dis Child 69:361-365

7. Voss LD et al (1992) Poor growth in school entrants as an index of organic disease: the Wessex growth study. BMJ 305:1400-1402
8. Hall JG et al (2007) Handbook of physical measurements. Oxford University Press, New York

9. Brook CG (1986) Turner syndrome. Arch Dis Child 61:305-309

10. Saenger $P$ et al (2001) Recommendations for the diagnosis and management of Turner syndrome. J Clin Endocrinol Metab 86:3061-3069

11. Ranke MB et al (2002) Adult height after GH therapy in 188 Ullrich-Turner syndrome patients: results of the German IGLU Follow-up Study 2001. Eur J Endocrinol 147:625-633

12. Ross J et al (2011) Impact of age and duration of growth hormone therapy in children with Turner syndrome. Horm Res Paediatr 76:392-399

13. Durand C et al (2010) Enhancer elements upstream of the SHOX gene are active in the developing limb. Eur J Hum Genet 18:527-532

14. Munns CF et al (2003) Familia growth and skeletal features associated with SHOX haploinsufficiency. J Pediatr Endocrinol Metab 16:987996

15. Niesler B et al (2007) The novel human SHOX allelic variant database. Hum Mutat 28:933-938

16. Rappold G et al (2007) Genotypes and phenotypes in children with short stature: clinical indicators of SHOX haploinsufficiency. J Med Genet 44:306-313
17. Chen J et al (2009) Enhancer deletions of the SHOX gene as a frequent cause of short stature: the essential role of a $250 \mathrm{~kb}$ downstream regulatory domain. J Med Genet 46:834839

18. Netchine I et al (2007) 11p15 imprinting center region one loss of methylation is a common and specific cause of typical Russell-Silver syndrome: clinical scoring system and epigenetic-phenotypic correlations. J Clin Endocrinol Metab 92:31483154

19. Bartholdi D et al (2009) Epigenetic mutations of the imprinted IGF2-H19 domain in Silver-Russell syndrome (SRS): results from a large cohort of patients with SRS and SRS-like phenotypes. J Med Genet 46:192-197

20. Eggermann T et al (2009) Broad clinical spectrum in Silver-Russell syndrome and consequences for genetic testing in growth retardation. Pediatrics 123:e929-931

21. Wollmann HA et al (1995) Growth and symptoms in Silver-Russell syndrome: review on the basis of 386 patients. Eur J Pediatr 154:958-968

22. Wakeling EL et al (2010) Epigenotype-phenotype correlations in Silver-Russell syndrome. J Med Genet 47:760-768
23. Toumba M et al (2010) Effect of long-term growth hormone treatment on final height of children with Russell-Silver syndrome. Horm Res Paediatr 74:212-217

24. Gicquel C et al (2005) Epimutation of the telomeric imprinting center region on chromosome 11p15 in Silver-Russell syndrome. Nat Genet 37:1003-1007

25. Noonan JA (1968) Hypertelorism with Turner phenotype. A new syndrome with associated congenital heart disease. Am J Dis Child 116:373-380

26. Lee DA et al (2005) Psychological profile of children with Noonan syndrome. Dev Med Child Neurol 47:35-38

27. Burgt I van der (2007) Noonan syndrome. Orphanet J Rare Dis 2:4

28. Dahlgren J (2009) GH therapy in Noonan syndrome: review of final height data. Horm Res 72(Suppl 2):46-48

29. Shiang R et al (1994) Mutations in the transmembrane domain of FGFR3 cause the most common genetic form of dwarfism, achondroplasia. Cell 78:335-342

30. Trotter TL, Hall JG (2005) Health supervision for children with achondroplasia. Pediatrics 116:771-783

31. Spranger J (1988) Bone dysplasia,families'. Pathol Immunopathol Res $7: 76-80$

\section{CME.springer.de wird zur e.Akademie}

Die e.Akademie von Springer Medizin ist die Weiterentwicklung von CME.springer.de und bietet Ihnen ein noch umfassenderes und moderneres Fortbildungsangebot: Mehr als 1500 Fortbildungsmodule, neue e.Learningformate und multimediale Elemente machen Ihre Fortbildung und das Sammeln von CME-Punkten noch flexibler und komfortabler.

e.CME: Als Zeitschriftenabonnent stehen Ihnen in der e.Akademie nach wie vor alle zertifizierten Fortbildungsbeiträge Ihrer Zeitschrift als e.CME zur Verfügung. Darüber hinaus haben Sie künftig die Möglichkeit, Beiträge Ihrer Zeitschrift, deren Zertifzierungszeitraum abgelaufen ist, weiterhin für Ihre Fortbildung und persönlichen Wissenscheck zu nutzen.

\section{- Der direkte Weg zur e.Akademie unter springermedizin.de/eAkademie}

Nutzer von e.Med, dem Springer Medizin Online-Paket, profitieren vom vollen Leistungsumfang der e.Akademie: Mehr als 1500 e.CMEs aus den wichtigsten medizinischen Fachgebieten werden ergänzt durch die neuen e.Learningformate e.Tutorial und e.Tutorial plus.

Das e.Tutorial ist speziell für die Online-Fortbildung konzipiert und didaktisch optimiert. Klar gegliederte Lernabschnitte, besondere Hervorhebung von Merksätzen, zoomfähige Abbildungen und Tabellen sowie verlinkte Literatur erleichtern das Lernen und den Erwerb von CME-Punkten.

Das e.Tutorial.plus bietet multimedialen Zusatznutzen in Form von Audio- und Videobeiträgen, 3D-Animationen, Experteninterviews und weiterführende Informationen. CME-Fragen und Multiple-Choice-Fragen innerhalb der einzelnen Lernabschnitte ermöglichen die Lernerfolgskontrolle.

\section{- Weitere Informationen zum e.Med-Komplettpaket und Gratis-Testangebot unter springermedizin.de/eMed}




\section{CME-Fragebogen}

\section{kostenfreie Teilnahme am e.CME für Zeitschriftenabonnenten}

Welche Aussage zur Fragestellung Kleinwuchs trifft nicht zu?

$\square$ SHOX-Deletionen und Mutation sind die häufigste bekannte genetische Ursache für ",idiopathischen" Kleinwuchs.

$\square$ Das Noonan-Syndrom geht in der Regel mit mittelgradiger Intelligenzminderung einher.

$\square$ Neben populationsspezifischen Perzentilenkurven stehen panethische Kurven der WHO zur Verfügung.

$\square$ Die Körperhöhe der Eltern ermöglicht die Berechnung der zu erwartenden Zielgröße eines Kindes.

$\square$ Die häufigsten Ursachen des Kleinwuchses sind Skelettdysplasien.

Welche Aussage zur Diagnosestellung von Kleinwuchs-Syndromen trifft nicht zu?

$\square$ Die Bestimmung der Körperhöhe und der Armspanne können einen Hinweis auf eine dysproportionierte Wachstumsverzögerung geben.

$\square$ Röntgenbilder, insbesondere der Hand, können auch bei proportioniertem Kleinwuchs diagnostische Hinweise geben.

Das Knochenalter ist ein Maß der Knochenreife.

$\square$ Die Familienanamnese kann Hinweise auf den Erbgang geben.

$\square$ Ein verzögertes Knochenalter bestätigt die Diagnose einer konstitutionellen Wachstumsverzögerung.

Welches klinische Zeichen ist nicht typisch für ein Ullrich-Turner-Syndrom?

$\square$ Tiefer, invertierter Haaransatz

$\square$ Pseudohydrozephalus

$\square$ Pterygium colli

$\square$ Brachymetakarpalie

$\square$ Hand- und Fußrückenödeme

Bitte beachten Sie:

- Teilnahme nur online unter: springermedizin.de/eAkademie

- Die Frage-Antwort-Kombinationen werden online individuell zusammengestellt.

- Es ist immer nur eine Antwort möglich.

? Defekte welcher der folgenden Gene gehen nicht mit Kleinwuchs einher?

$\square$ NSD1

$\square$ SHOX

$\square$ FGFR3

$\square$ PTPN11

SHOC2

Ein 12 Monate alter Junge wird in der Sprechstunde vorgestellt zur Abklärung von Kleinwuchs und Gedeihstörung. Wegen Ernährungsschwierigkeiten erfolgt eine Sondenernährung. Die klinische Untersuchung ergibt einen relativ großen Kopf mit dreieckiger Gesichtsform mit prominenter Stirn und eine Körperasymmetrie. Welchen Befund würden Sie am ehesten erwarten?

$\square$ Eine Deletion der Prader-Willi-Region $15 q 11.2-q 13$

$\square$ Eine SHOX-Punktmutation

$\square$ Eine paternale Hypomethylierung des IC1 in 11p11.5

$\square$ Eine UPD14

$\square$ Eine PTPN11-Mutation

Welche Aussage zum Leri-Weill-Syndrom trifft zu?

$\square$ Eine Madelung-Deformität liegt in der Regel bei Geburt vor.

$\square$ Charakteristisch ist eine Rhizomelie der Extremitäten.

$\square$ Die Ursache sind homozygote Deletionen des SHOX-Gens.

Deletionen außerhalb der codierenden Region des SHOX-Gens können die Ursache sein.

$\square$ Die Sequenzierung des SHOX-Gens identifiziert die häufigsten Ursachen.

Welche Aussage zur Achondroplasie trifft nicht zu?

Bei 98\% der Patienten lässt sich eine rekurrente Mutation im FGFR3-Gen nachweisen.
Ursächlich sind rekurrente Mutationen im FGFR2-Gen.

Sie ist mit der Hypochondroplasie und der thanatophoren Dysplasie Teil einer allelischen Krankheitsgruppe.

Sie gehört zu den häufigsten Skelettdysplasien.

$\square$ Die Verengung des Foramen magnums kann zu neurologischen Symptomen führen.

Welchen Befund erwarten Sie typischerweise nicht bei einem Noonan-Syndrom?

$\square$ Pulmonalstenose

Lymphangiektasien

Erhöhte Nackentransparenz im ersten Trimenon

$\square$ Trichterbrust

Epilepsie

Zu den für ein Ullrich-Turner-Syndrom typischen Fehlbildungen zählt nicht:

$\square$ Pulmonalstenose

$\square$ Aortenisthmusstenosen

$\square$ Bikuspide Aortenklappe

$\square$ Nierenaplasien

Hufeisennieren

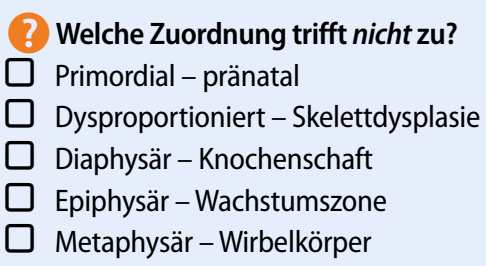

Diese zertifizierte Fortbildung ist 12 Monate auf springermedizin.de/ eAkademie verfügbar.

Dort erfahren Sie auch den genauen Teilnahmeschluss. Nach Ablauf des Zertifizierungszeitraums können Sie diese Fortbildung und den Fragebogen weitere 24 Monate nutzen. 